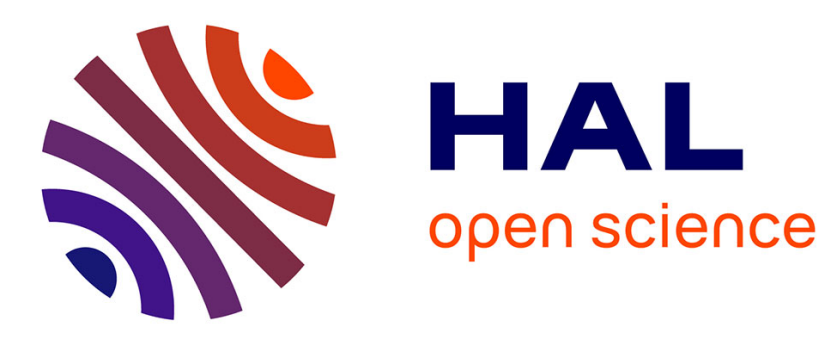

\title{
On image analysis and micromechanics
}

\author{
Dominique Jeulin
}

\section{To cite this version:}

Dominique Jeulin. On image analysis and micromechanics. Revue de Physique Appliquée, 1988, 23

(4), pp.549-556. 10.1051/rphysap:01988002304054900 . jpa-00245801

\section{HAL Id: jpa-00245801 https://hal.science/jpa-00245801}

Submitted on 1 Jan 1988

HAL is a multi-disciplinary open access archive for the deposit and dissemination of scientific research documents, whether they are published or not. The documents may come from teaching and research institutions in France or abroad, or from public or private research centers.
L'archive ouverte pluridisciplinaire HAL, est destinée au dépôt et à la diffusion de documents scientifiques de niveau recherche, publiés ou non, émanant des établissements d'enseignement et de recherche français ou étrangers, des laboratoires publics ou privés. 


\title{
On image analysis and micromechanics
}

\author{
D. Jeulin
}

\author{
Centre de Géostatistique, Ecole des Mines de Paris, 35 Rue Saint-Honoré, \\ 77305 Fontainebleau, France \\ and Centre des Matériaux, B.P. 87, 91003 Evry Cedex, France
}

(Reçu le 9 septembre 1987, accepté le 9 novembre 1987)

\begin{abstract}
RESUME - Nous montrons par des exemples comment une analyse morphologique quantitative de microstructures peut contribuer à développer une approche micromécanique dans 1 'étude du comportement mécanique des matériaux.

* Dans la première partie, nous rappelons les principaux paramètres morphologiques qui donnent une description quantitative d'un point de vue statistique : fonctions de corrélation $d$ 'ordre $n$, moments $Q(B)$. Ces paramètres peuvent être mesurés sur des matériaux par analyse d'images, ou calculés pour certaines classes de modèles aléatoires de structures, afin de résumer les données disponibles sur la microstructure.

* Lorsqu'on étudie les propriétés mécaniques, il peut être utile de développer des mesures spécifiques. Une illustration en est donnée à travers trois exemples dans le domaine de la mécanique de la rupture :

- une approche "auto-cohérente" a permis de relier la résistance mécanique du coke à sa structure poreuse ;

- les associations spatiales entre fissures et structure minéralogique ont été caractérisées à 1'aide de mesures appropriées, dans 1'étude de la dégradation d'un aggloméré de minerai de fer au cours de sa réduction;

- des simulations par analyse d'image de la propagation de fissures dans du graphite poreux ont été développées pour expliquer le rôle des macropores intergranulaires sur 1'anisotropie de ténacité et de résistance mécanique.
\end{abstract}

ABSTRACT - We give an illustration of how quantitative morphological analysis of microstructures may help to develop a micromechanical approach to investigate the mechanical behaviour of materials.

* In the first part, we recall the main morphological parameters that account for a quantitative description of microstructures from a statistical point of view : correlation functions of order $\mathrm{n}$, moments $\mathrm{Q}(\mathrm{B})$. These parameters may be measured on materials by means of image analysis, or may be calculated for certain classes of random models of structures, in order to sum up available data on the microstructure.

* When studying mechanical properties, it may be useful to develop specific measurements. This will be illustrated by three examples in the case of fracture mechanics :

- a "self-consistent" approach allowed to relate coke strength to its porous structure ;

- spatial relationship between crack paths and underlying mineralogical structure were looked for with appropriate measurements in a study of iron ore sinter degradation during reduction; - image analysis simulations of crack propagation in porous graphite were developed to explain the role of intergranular macropores on toughness and strength anisotropy.

\section{INTRODUCTION}

The use of a microstructural description to explain the mechanical behaviour of materials is necessary, and rather widespread, mainly on a qualitative basis : one of the most common examples is the diagnosis of the failure of materials from fractogra phic examinations, where "defects" in the material structure are detected in order to point out the cause of the fracture process.

Getting quantitative data on the structure of materials may help to advance further in the determi- nation of relationships between overall behaviour and microstructure. Staying in the field of fractography, quantitative fractography performed by means of image analysis [1] allows getting useful and reliable data.

From image analysis, it is also possible to measure microstructural parameters that can be used in a "micro-mechanical" model, i.e. a model using microstructural information to predict the mechanical behaviour. This point is illustrated by several examples : 
- in section 2, the use of a statistical approach for the analysis of microstructures by means of correlation functions and functional moment $Q(B)$ is recalled;

- in section 3, the development of specific morphological measurements for micromechanics is discussed, in the frame of fracture mechanics of brittle porous heterogeneous materials : first the fracture strength of a porous material is considered ; then two morphologica1 approaches of damage during fracture are proposed.

\section{STATISTICAL APPROACH OF MICROSTRUCTURES}

2.1 Definition of Statistical Spatial Properties Intrinsic heterogeneities of the structure

a
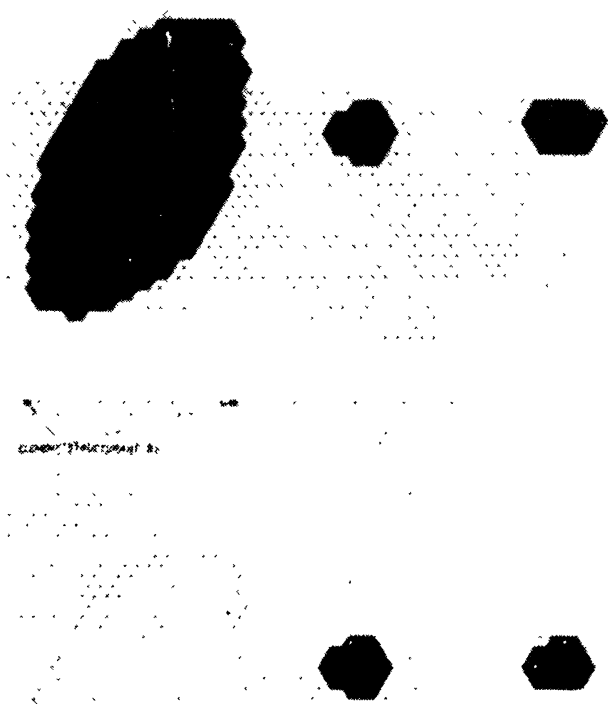

From (1) it is obvious that the use of different structuring elements (with different shapes, as illustrated in figure 1, or of different sizes) give a complete description of the morphological information contained in the microstructure. Particular choices of $B$ give specific morphological data. A classification of the types of structuring elements (and operations derived from erosion) results in various morphological criteria [6].

In the case of multiphase materials (phase $x_{1}, \ldots$ $x_{k}$ ), it is possible to consider a finite set of points $x, x+h_{1}, \ldots x+h_{n}$ as a structuring element $B$, and to define

$$
\begin{gathered}
\left.T_{1,2}, \ldots k^{(x, h}, \ldots h_{n}\right)= \\
P\left\{x \in x_{1}, x+h_{1} \in x_{2} \ldots x_{n} \in x_{k}\right\}= \\
P\left\{x \in x_{1} \cap x_{2-h} \cap \cdots \cap x_{k-h}\right\}
\end{gathered}
$$

Relation (2) gives n-points merphological correla- of materials result in spatial variations of their physical properties at different scales : it is the case of the optical microscope level for the polycrystal structure of steel, and the multiphase mineralogical structure of iron ore sinters [2]. A correct approach of the quantitative morphological analysis of such materials is provided by random sets modelling in the frame of mathematical morphology $[2-8]$. It can be shown that a random set $X$ is completely characterized from its functional moments $Q(B, x)[3,4]$ :

$$
\mathrm{Q}(\mathrm{B}, \mathrm{X})=\mathrm{P}\left\{\mathrm{Bx} \subset \mathrm{X}^{\mathrm{C}}\right\}=\mathrm{P}\left\{\mathrm{x} \in \mathrm{X}^{\mathrm{C}} \odot \stackrel{\mathrm{B}}{ }\right\}
$$

where $\mathrm{X} \Theta \check{\mathrm{B}}$ is the morphological operation (acting on $\mathrm{X}$ ) called erosion, by the structuring element $\mathrm{B}$, as illustrated in figure 1.

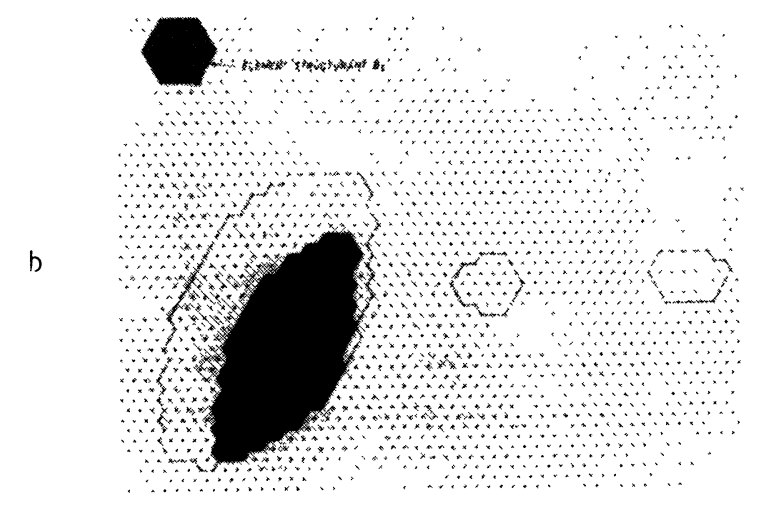

Figure 1 - Example of erosion $\mathrm{X} \Theta \breve{\mathrm{B}}$

a. Initial image $X$ (in black)

b. $X \ominus H(r) \quad\left(H^{\prime}(r)=\right.$ hexagon $)$

c. $\mathrm{x} \Theta_{\mathrm{h}}$ (erosion by a pair of points $x, x+h)$

tion functions $\left[5,7^{-}\right]$, to study order $\mathrm{n}$ mutual associations of phases. Particular cases are given by $\mathrm{n}=2$ (covariance análysis in fig. 1-c) and by $\mathrm{n}=3$ (third order correlation functions).

From the n-points morphological correlation functions, it is possible to calculate the correlation functions of the material physical properties $a(x)$, provided that the individual properties $a_{i}$ of each phase of the material are known [7] :

- second order correlation function $W(x, x+h)=$

$\sum_{i, j} a_{i} a_{j} C_{i j}(x, x+h)$ (where $C_{i j}(x, x+h)=P\left\{x \in x_{i}\right.$; $\left.\left.x+h \in x_{j}\right)\right\}$ is the cross - covariance between phases $x_{i}$ and $x_{j}$ ).

- third order correlation function

$$
\begin{gathered}
\mathrm{W}\left(\mathrm{x}, \mathrm{x}+\mathrm{h}_{1}, \mathrm{x}+\mathrm{h}_{2}\right)= \\
\Sigma a_{i} a_{j} a_{k} \mathrm{~T}_{i j k}\left(\mathrm{x}, \mathrm{x}+\mathrm{h}_{1}, \mathrm{x}+\mathrm{h}_{2}\right)
\end{gathered}
$$




\subsection{Determination of Statistical Spatial Proper- ties.}

In most cases, considered materials are, on average, homogeneous in space. It means that they can be modelled by stationary ergodic random sets. Therefore the functional moments and correlation functions in equations (1) to (4) do not depend on the location of point $x$ in the material, and a11 the corresponding probabilities can be estimated from volume fraction measurements.

Practically, the functional and the statistical morphological moments can be determined in two different ways [7] :

- for a given material, they can be estimated from measurements on samples by image analysis with appropriate image transformations (erosions in (1) or translations and intersections in (2)) and votume fraction (or area fraction on plane sections) measurements ;

- they can be calculated in a theoretical way for a given model of structure as a function of the model parameters. This is mainly useful for high order moments, which are difficult to estimate, or even inaccessible when 3-D structuring elements are required (e.g. 4th order correlation functions) and only planar samples are available. Examples are detailed in [7] and summed up in [8].

\subsection{Use of Correlation Functions of Material Properties.}

The correlation functions can be used as quantitative descriptors of the microstructure, to sum up morphological information (for instance, the changes of the covariance function with the distance $h$ reflect the scales in the material).

They also enter as parameters in the prediction of effective (macroscopic) properties of materials from microscopic information in the case of linear relations (such as linear elasticity for mechanical behaviour). Representative contributions to this approach can be classified according to different methods :

- statistical theory of heterogeneous materials $[9,10]$.

- perturbation theory $[11,12,13]$.

- bounds derived from variational methods $[14,15$, $16]$.

They all lead to an optimal estimation of effective properties up to order $n$ (which is the order of the correlation functions entering into the approximation), and are limited in the applications to nonporous materials.

So, it is possible either by image analysis or by random structure modelling, to use the available statistical theories to estimate macroscopic linear properties of materials from their microstructure.

\section{SPECIFIC MORPHOLOGICAL MEASUREMENT IN THE CASE OF FRACTURE MECHANICS.}

There is presently no general theoretical statistical approach to predict fracture mechanics parameters from microscopical information. Solving this problem is difficult mainly for porous materials, where the dispersion of stresses in the material (due to stress concentration) is so high that approximate methods such as calculations based on perturbations are irrelevant. Instead of general methods, particular prediction (based on simplified assumptions of the geometry of the material) can be proposed as is illustrated in this paper for coke material.
As far as damage to a material (i.e. microcracking under stress) is concerned, new morphological measurements can also be introduced (section 3.2).

\subsection{Relation between tensile strength and pore size of porous materials.}

An example of porous material is given by metallurgical coke (Figure 2), that is used in the blast furnace. Its pore volume fraction is nearly $50 \%$. The sizes of pores range from $10 \mu \mathrm{m}$ to several $\mathrm{mm}$. Its tensile strength may be estimated from diametral compression tests on cylinders drilled in the material. A "self-consistent" approach was proposed to relate coke tensile strength to its porous structure $[5,17,18]$. In this approach, an equivalent medium with a simplified porous geometry and an homogeneous matrix is considered. Two different mechanical assumptiors can be used.

- classical fracture mechanics can be applied to the largest pores in the material sample, considered as an isolated flaw inside an homogeneous medium.

-fracture mechanics calculation on a periodic porous structure can provide an estimation of the tensile strength as a function of the critical stress intensity factor $\mathrm{K}_{\mathrm{I}}$ of the matrix. It accounts for mechanical interactions between pores but it neglects pore size distribution.

\subsubsection{Prediction of the tensile strength} from the size of the largest pore.

From classical fracture mechanics, the tensile strength $\sigma_{c}$ of an homegeneous material containing an isolated crack (with length 2a) is expressed by :

$$
\sigma_{c}=\sqrt{\frac{G_{\text {Ic }} E}{\text { II }\left(1-v^{2}\right) a}}
$$

where $\mathrm{E}, \mathrm{V}$, and $\mathrm{G}_{\mathrm{Ic}}$ are Young modulus, Poisson coefficient and toughness of the homogeneous material. For a porous material (with inhomogeneous pore size distribution) it is possible to use (5), where a is a "size parameter" of the largest pore, and where the other parameters depend on pore morphology.

The Poisson coefficient is usually considered as constant (for a given matrix), while empirical formulations were proposed to relate Young's modulus [19] and toughness [20] to pore volume fraction $\mathrm{v}_{\mathrm{v}}:$

$$
\begin{aligned}
E & =E_{0} \exp \left(-b V_{v}\right) \\
G_{I c} & =G_{0} \exp (-\alpha V v)
\end{aligned}
$$

From equations (5), (6), (7), a relation between tensile strength $\left(\sigma_{c}\right)$, pore volume fraction, and the average size of the largest pore (I) is derived :

$$
\sigma_{\mathrm{c}}=\frac{\mathrm{K}}{\sqrt{\mathrm{I}}} \exp \left(-\beta \mathrm{V}_{\mathrm{v}}\right)
$$

3.1.2 Prediction of the tensile strength from mechanical interactions in a regular array of spherical pores.

Finite element calculations allowed proposing a different formulation to relate strength to pore geometry [2i] : spherical pores with radius a are implanted on an hexagonal grid. For a tensile stress applied orthogonally to the grid and an homogeneous matrix (with critical stress intensity factor $\mathrm{K}_{\mathrm{Ic}}$ ), an approximate equation relating $\sigma_{c}$ 
to the other parameters is given by

$$
\sigma_{c}=K_{\text {Ic }} \frac{\sqrt{\pi}}{2 \sqrt{a}} \frac{\sqrt{\pi / 2 v_{v}}}{\sqrt{\tan \left(\frac{\pi}{2} v_{v}\right)}}
$$

It is possible to replace a real porous structure (with pore volume fraction $\mathrm{V}_{\mathrm{v}}$ and specific surface area $\mathrm{S}_{\mathrm{v}}$ ) by an equivalent periodic structure. Therefore $\mathrm{V}$

$$
\sigma_{c}=\frac{\Pi 3 / 2}{8} K_{\text {Ic }} \sqrt{\frac{S_{v}}{\tan \left(\frac{\Pi}{2} v_{v}\right)}}
$$

The two approaches were tested on different metallurgical cokes $[5,17,18]$. The morphological parameters entering into equations (8) and (10) were measured by image analysis on polished sections prepared close to the fracture plane of the samples. Each of them was explored by 4 fields of measurement of size $\left(4,86 \times 4,2 \mathrm{~mm}^{2}\right)$, where pores were filtered according to their size, by hexagons (of $95 \mu \mathrm{m}, 190 \mu \mathrm{m}, 285 \mu \mathrm{m}, 380 \mu \mathrm{m}$ and $475 \mu \mathrm{m}$ sides (Figure 3)). Pore volume fractions and pore average intercept I were measured for each size of the filter. They were averaged for the 4 fields and for samples belonging to the same class of tensile strength. Details of the result are given in the references. Here we can only report the conclusions : in the case of coke material, the approach derived from relation (8) (initiation of fracture on the largest pores) gives the best relation strength-porosity.

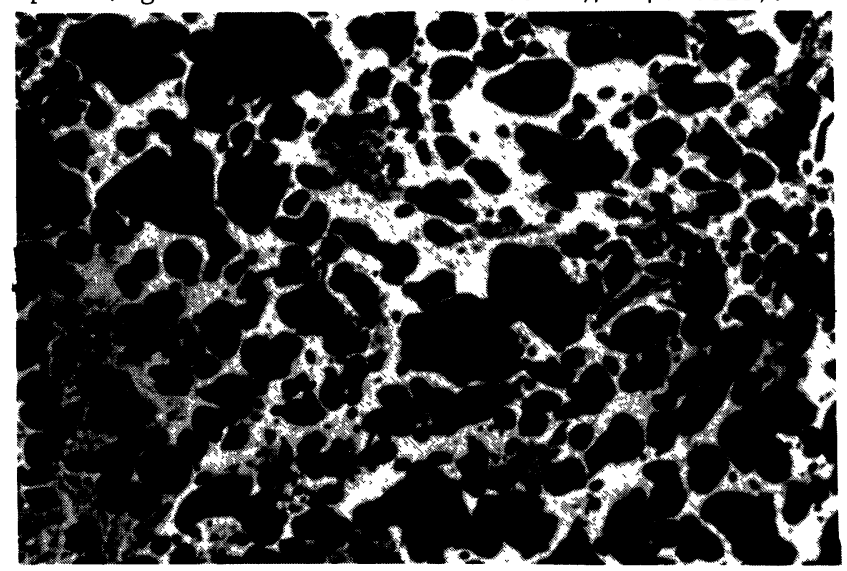

Figure 2. Porous structure of metallurgical coke (pores in black, and carbon phase in grey).
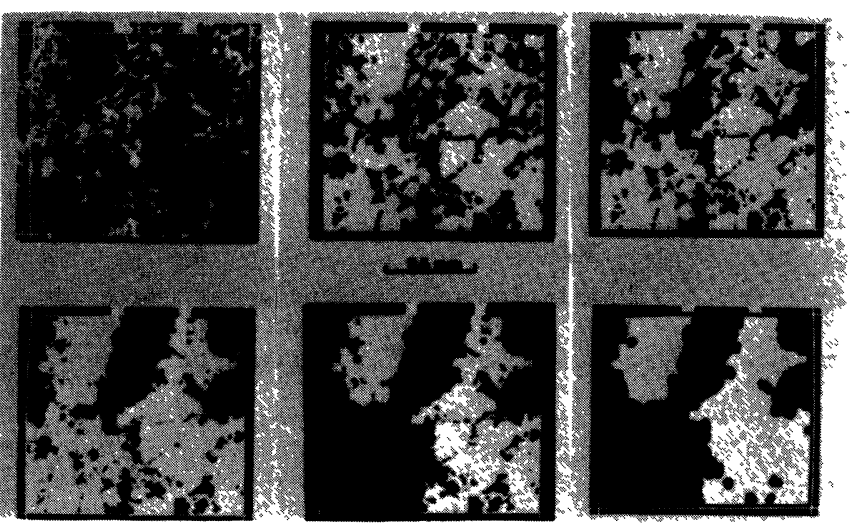

Figure 3. Détection of largest pores seen in a section of a coke sample (see text).
It allows deriving an estimation of parameters $\beta$ and $K$. The first one is a pore shape parameter, while the second one is related to the critical stress in tensity factor of the carbon matrix. It is therefore possible to estimate porosity-free mechanical properties of the material.

This approach is worth being followed for other materials. It should also be of interest to introduce more realistic geometries of porous media and more appropriate relations than (6) and (7) between mechanical properties and porosity.

\subsection{Morphological Approach of the Development of} Damage During Material Fracture.

The purpose of this section is to show how a morphological and image analysis approach may be used to study spatial interactions during crack propagation in materials. Two different cases are considered :

- morphological analysis of the microcracks

- image analysis simulation of crack propagation.

3.2.1 Morphological analysis of microcracking in multi-component materials.

When studying a cracked material, it may be useful to obtain statistical information on the 1ocation of the crack network (and of its characteristics such as end points, multiple points...) with respect to the microstructure. It should be noted that in the case of cracks with zero thickness, the use of covariances or of correlation functions as presented in section 2, would fail, as these functions are identically reduced to zero.

Thus, we developed appropriate methods for the quantitative study of spatial distributions in multi-component structures $[22,2]$ based on the elementary morphological operation called dilation ( $\rightarrow$ )

$$
X \oplus B=\left(X^{c} \Theta B\right)^{c}
$$

One of the components ( $\mathrm{Xk}$, namely a crack in figure 4) is selected. From this component, neighbourhoods with increasing sizes (by dilations) are
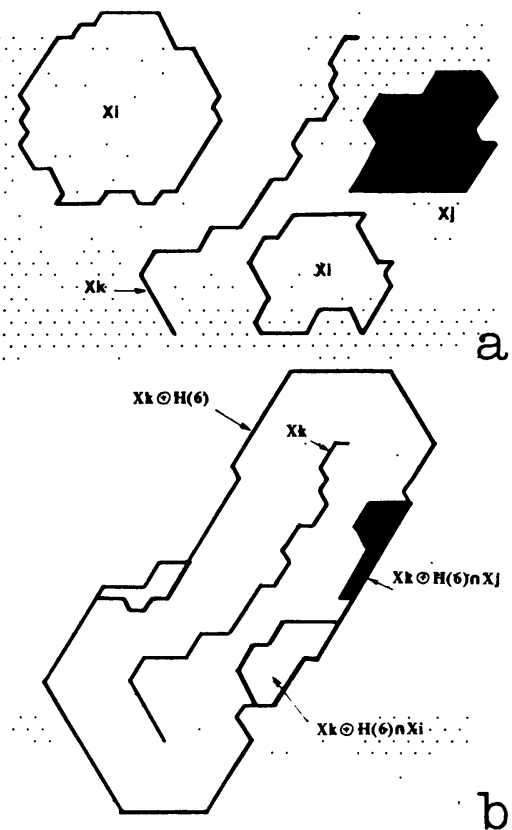

$\mathrm{b}$

Figure 4 - Principle of measurement of crack (Xk) preferential path in a multi-component material $\left(x_{i}, x_{j}\right)$ : dilation by an hexagon of
size 6$).$ 
built, which are used as selected fields of measurements to study the other components $\left(X_{i}\right)$ of the structure.

For instance, using a dilation by an hexagon $r, H(r)$, one can define

$$
\Phi_{j}(k, r)=v_{v}\left[x_{k} \oplus H(r) \cap x_{j}\right]
$$

and the ratio

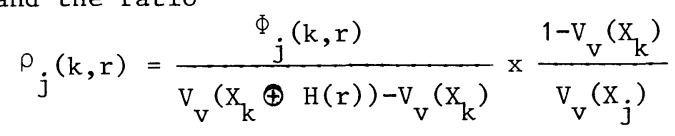

When $\rho_{j}(k, r)>1$, a preferential spatial association (at scale $r$ ) between phases $k$ and $j$ may be suspected. When $\rho_{j}(k, r)<1$, a repulsion effect (at scale $r$ ) of $x_{k}$ by ${ }^{j} x_{j}$ is put forward.

The functions $\Phi_{j}^{j}(k, r)$ and $\rho_{j}(k, r)$ are related to the distribution functions of distances :

- distance of a random point in $x_{k}^{c}$ to $x_{k}$ boundary

$$
\begin{aligned}
\mathrm{T}(\mathrm{k}, \mathrm{r}) & =\mathrm{P}\left\{\mathrm{d}\left(\mathrm{x}, \mathrm{x}_{\mathrm{k}}\right)<\mathrm{r} \mid \mathrm{x} \in \mathrm{x}_{\mathrm{k}}^{\mathrm{c}}\right\} \\
& =\frac{\mathrm{V}_{\mathrm{V}}\left(\mathrm{X}_{\mathrm{k}} \oplus \mathrm{H}(\mathrm{r})\right)-\mathrm{V}_{\mathrm{v}}\left(\mathrm{x}_{\mathrm{k}}\right)}{1-\mathrm{V}_{\mathrm{V}}\left(\mathrm{x}_{\mathrm{k}}\right)}
\end{aligned}
$$

- distance of a random point in $\mathrm{x}_{\mathrm{j}}$ to the boundary of $\mathrm{X}_{\mathrm{k}}$ :

$$
\begin{aligned}
T_{j}(k, r) & =P\left\{d\left(x, x_{k}\right)<\mid r x \in X_{j}\right\} \\
& =\Phi_{j}(k, r) / V_{v}\left(x_{j}\right) \\
\rho_{j}(k, r) & =T_{j}(k, r) / T(k, r)
\end{aligned}
$$$$
\text { and }
$$

An application of this method concerns the examination of crack propagation in multiphase iron ore sinters at the beginning of its reduction in the blast furnace $[22,23]$. Examples of microcracks developed in this material, sometimes resulting in its catastrophic destruction [5] are shown in $\mathrm{fi}$ gure 5. The microcracks are due to local swelling of hematite when it is reduced into porous magnetite at low temperature.

The crack path was studied by image analysis, from estimation of mentioned functions $\Phi_{j}(k, r)$, using dilations of cracks by hexagons of increasing side $\mathrm{r} H(\mathrm{r})$, and area fraction measurements.

The results obtained from measurements on 3000 fields of $1 \mathrm{~mm}^{2}$ area are reported in figure 6 :

- the distribution of distances from a point of the material to cracks (figure 6a) gives a good evaluation of the intensity of cracking : for instance the use of the median distance (which decreases when cracking increases) allows measuring an overal1 damage parameter in different sinters [23].

- the volume fraction of phases (excluding pores in the present case) in neighbourhoods of cracks with increasing sizes is reported in figure $6 \mathrm{~b}, \mathrm{c}$. It can be concluded that in this material cracks are mainly located into hematite (primary and secondary) and into slag, while they avoid magnetite, and mainly ferrites.

More details on the microcracking process may be obtained by measuring the same parameters at different steps of the damage progression in the material. Particular zones of the crack network (e.g. crack ends to study crack arrest ; crack initiation sites ; crack bifurcations ...) may be located with respect to the microstructure in a similar way $[2,22]$.

\subsubsection{Simulation by .image analysis of crack propagation.}

The preceding section was devoted to the definition and the application of morphological descriptors of the cracking process at a given
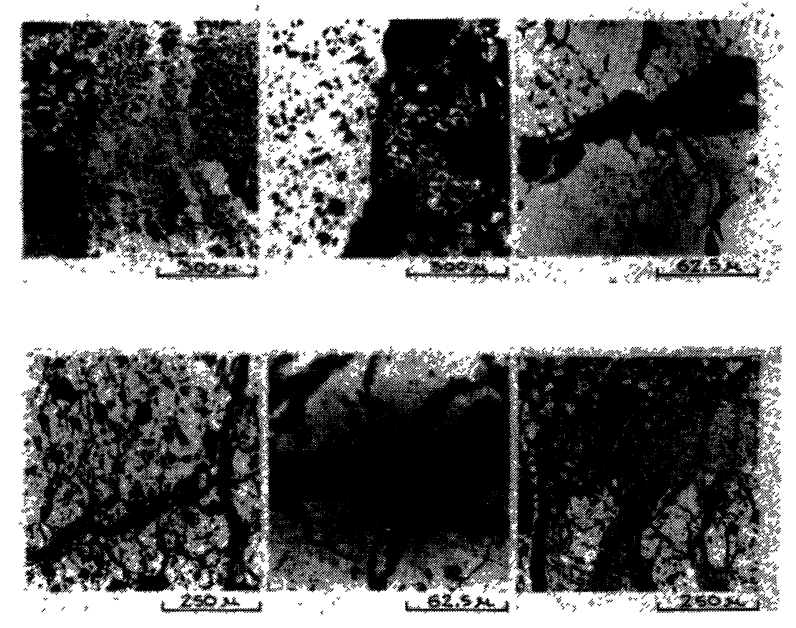

Figure 5. Examples of microcracking of iron ore sinters during the beginning of their reduc tion in the blast furnace conditions (laboratory test).

stage of its advance in the material. Their imple-mentation is made on samples after partial fracture. We are now concerned with an inveise process : how to predict a crack path in a material given the crack starting point, and given images of the microstructure ? A solution to this problem is proposed and tested in the case of a porous brittle material, polycrystalline graphite. It allows relating fracture toughness of the material, Gc, to a morphological parameter accounting for the simulated crack path length. Hereunder we sum up the approach, which is detailed elsewhere $[2,24$ and D. Jeulin, C. Larquetaux, P. Osmont "Simulation of crack propagation in a porous material by Image Analysis", to be published in Acta Stereologica] :

- polycrystalline graphite of arc furnace electrodes in a porous material (pore volume fraction $\approx$ $20 \%$ ), with grain size ranging from $1 \mathrm{~mm}$ to more than $10 \mathrm{~mm}$. The material contains intergranular macroporosity with $2-3 \mathrm{~mm}$ pore size. Due to the anisotropy of the process during electrode elaboration, there is an anisotropic distribution of grains, and therefore of intergranular pores in the material, resulting in anisotropic mechanical properties. This is illustrated by average results obtained from flexion tests, reported in Table 1 : 


\begin{tabular}{|c|c|c|c|c|}
\hline \multirow{2}{*}{ Orientation } & $(* *)$ & \multicolumn{2}{|c|}{$(* *),(* * *)$} & \multirow{2}{*}{$\begin{array}{l}\mathrm{G}_{\mathrm{c}}^{(* * *)} \\
\mathrm{J} / \mathrm{m}^{2}\end{array}$} \\
\hline & $\mathrm{E}(\mathrm{GPa})$ & $\begin{array}{c}\sigma_{R} \\
(\mathrm{M}+\mathrm{Ya}) \\
\end{array}$ & $\begin{array}{l}\mathrm{K}_{\mathrm{c}} \\
(\mathrm{MPa} \vee \mathrm{m})\end{array}$ & \\
\hline & 2, & 13,7 & 0.738 & 195, \\
\hline$\theta$ & 3,1 & 13,5 & 0.806 & 207,9 \\
\hline $\mathrm{Z}, \mathrm{R}$ & 4,2 & 18,1 & 0.908 & 228,2 \\
\hline
\end{tabular}

TABLE 1 - Anisotropy of polycrystalline graphite mechanical properties.

* the samples are identified in cylindrica1 coordinates (stress direction, crack propagation direction)

** sample size : $15 \times 15 \times 60 \mathrm{~mm}^{3}$

$* * *$ precracked specimen $(\mathrm{ao}=10 \mathrm{~mm})$ : $80 \times 15 \times 20 \mathrm{~mm}^{3}$.

- From fractographic examination, it is clear that intergranular fracture is dominant in this material, and that fracture is preferentially initiated on intergranular macropores (the critical flaw size deduced from fracture mechanics and from $\sigma_{R}$ and $K_{c}$ ranges from 1 to $2,5 \mathrm{~mm}$ ).

The results give a clue to generate 2-D simulations of the crack path in the material.

- The crack propagation simulation is based on an energy criterion : it is assumed that the actual fracture path will minimize the fracture energy consumption. If we consider an homogeneous material with pores and with effective fracture surface energy $\gamma_{S}$ for the matrix, the fracture work of the ma-

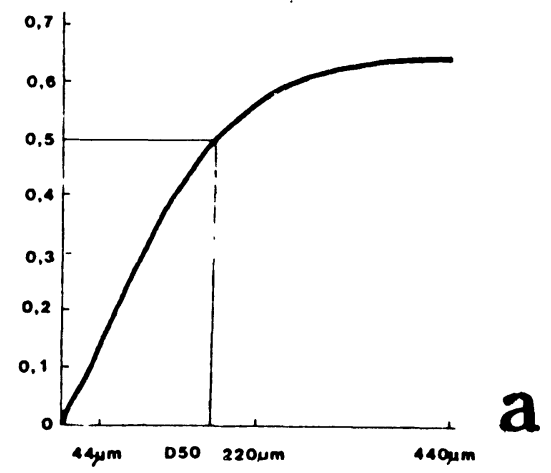

$\operatorname{Vv}\left(\right.$ CRACKS $\left.\oplus H(r) \cap X_{i}\right)$ (SOLID PHASE)

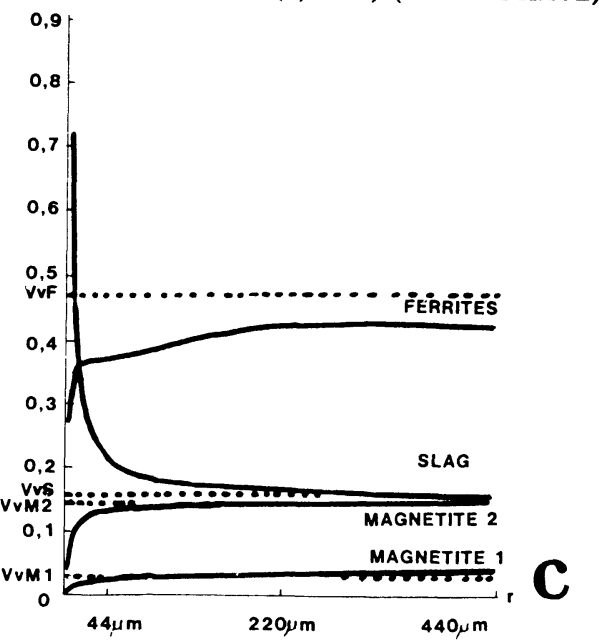

terial, when creating the total fracture surface $S$ is

$$
\mathrm{W}=2 \mathrm{~s} \gamma_{\mathrm{s}} \mathrm{e}
$$

with $e=\frac{\text { area of cracks in the matrix }}{S}$

Therefore, the effective toughness $G_{c}$ and stress intensity factor $\mathrm{K}_{\mathrm{c}}$ of the porous material are given by

$$
G_{c}=\frac{W}{S}=2 \gamma_{s} e=\frac{K_{c}^{2}}{E}
$$

with $G_{C}<$ toughness of the matrix.

A prediction of $G_{c}$ (from e) and fracture paths can be obtained by looking for paths giving e minima1 ("minimal path") in order to satisfy the criterion of minimal energy consumption.

This can be obtained by an image analysis algorithm, based on purely geometrical considerations (no stress distribution calculation is required in this method). The principle of this algorithm is as follows (the details are given in the above mentioned reference):

- in the case of an homogeneous medium submitted to a uniform tensile stress $\sigma$, the minimal path connecting the crack tip to the end of the sample is a straight line orthogonal to $\sigma$. It corresponds to the Euclidean distance between the crack tip and the sample end.

\section{$\operatorname{Vv}($ CRACKS $\oplus H(r) \cap$ Xi) (SOLID PHASE)}

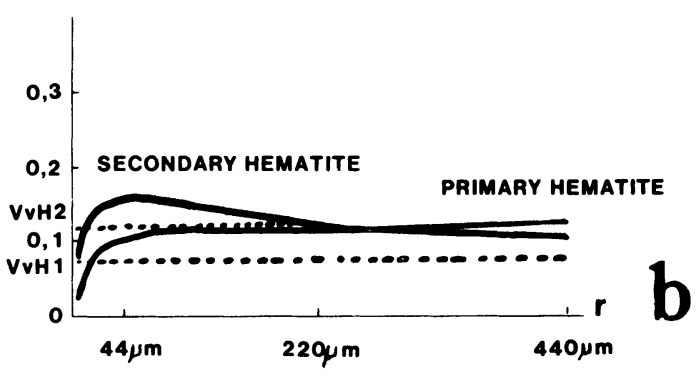

Figure 6. Example of quantitative study of the location of microcracks in a sinter material.

(a) distribution of distances from a point of the material to cracks.

(b) (c) Location of cracks with respect to the mineralogical components. 
- in the case of an heterogeneous medium (porous, or multiphase material), the distance must be valued according to the fracture surface energy of its components : for a porous medium $(\gamma=0$ in pores) we can put $\mathrm{d}(\mathrm{x}, \mathrm{y})=0$ for $\mathrm{x}$ and $\mathrm{y}$ in the same connected pore ; for hard particles $(\gamma=\infty)$, we can put $d(x, y)$ $=\infty$ for two points in the same particle.

In both situations the Euclidean distance is changed into a geodesic distance [25].

Crack propagation simulation is obtained by finding the geodesic paths $\mathrm{C}(\mathrm{x}, \mathrm{Y})$ connecting the crack tip $x$ to the end of the sample $Y$.

The two extreme cases $(\gamma=0$ for pores and $\gamma$ $=\infty$ for hard particles) are illustrated in figure 7 , where it is clear that in order to minimize the "effective" path length, the path will preferential1y go through pores or will avoid very hard particles. The final crack path may also be considered as a "percolating path" connecting a given point of the material to one of its edges.

For a porous material, we can estimate the morphological parameter e (named geodesic deviation) from

$$
\mathrm{e}=\frac{\mathrm{L}(\mathrm{C}(\mathrm{x}, \mathrm{Y}))}{\mathrm{d}(\mathrm{x}, \mathrm{Y})}
$$

This was done on graphite polished sections obtained from the cracked samples used in the experimental determination of $\mathrm{F}_{-}$' and $\mathrm{K}_{\text {- ( }}$ (see Table 1) by flexion tests. The simulations were performed with a LEITZ-TAS image analysis system on a $9,6 \times 9,6$ num $^{2}$ field [24], starting from the trace of the actual crack, and respecting the orientation of the structure with regard to the applied stress.

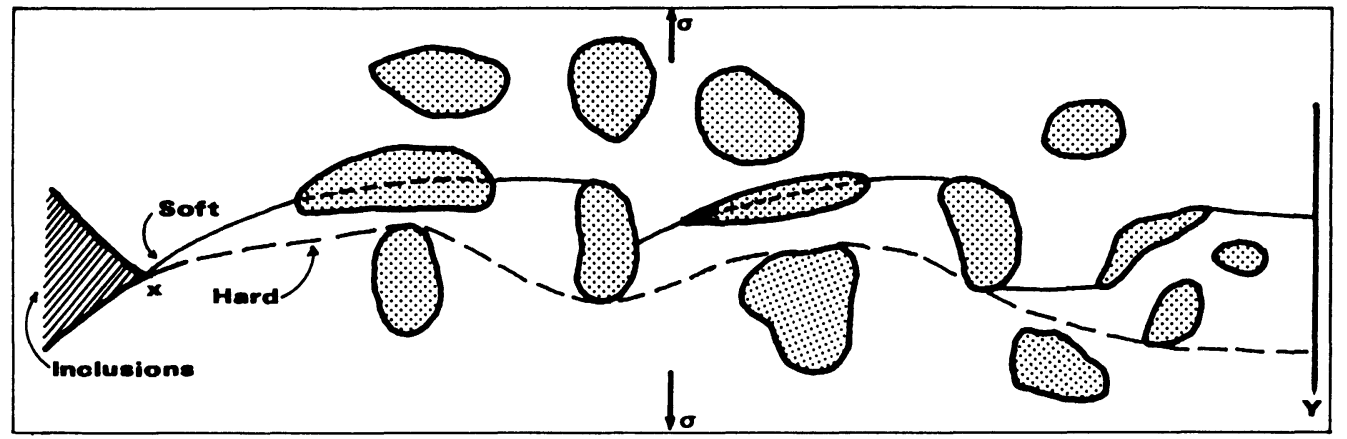

Figure 7 - The geodesic paths connecting $x$ to $Y$ for inclusions in two extreme cases $(\gamma=0$ and $\gamma=\infty)$.

The method is valided by the results presented in

figures 8 and 9 .
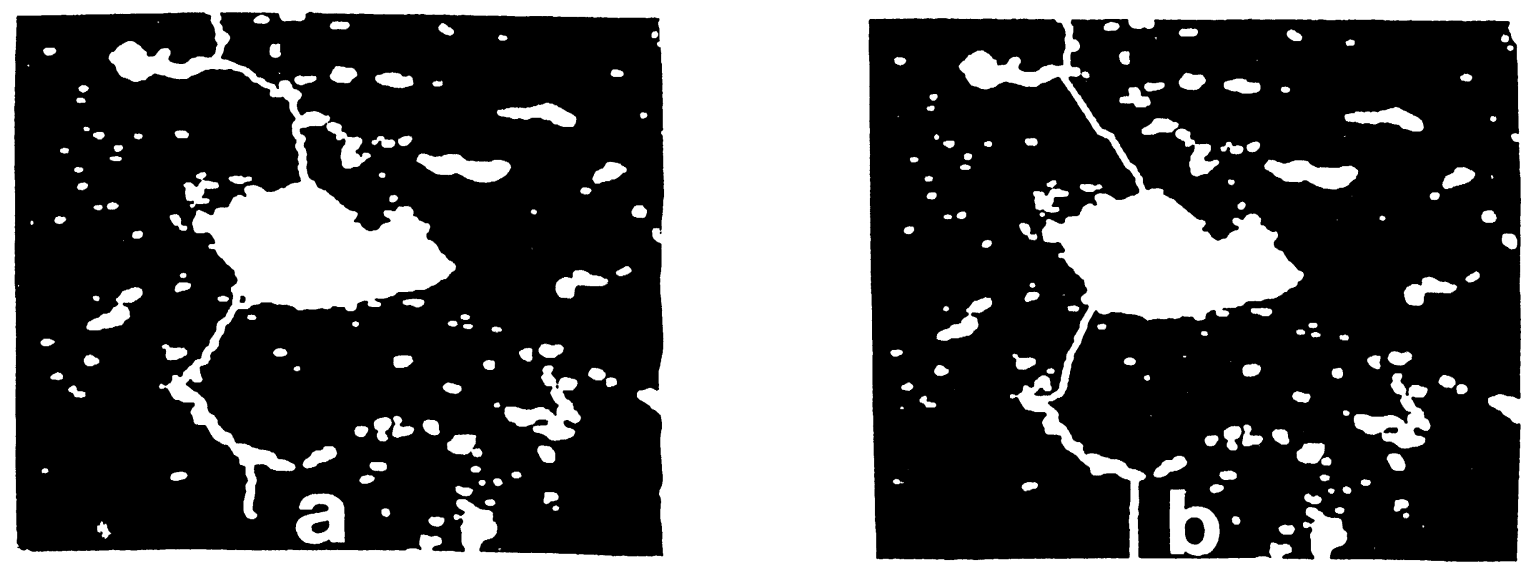

Figure 8 - Comparison between actual crack (a) and simulated crack (b). 

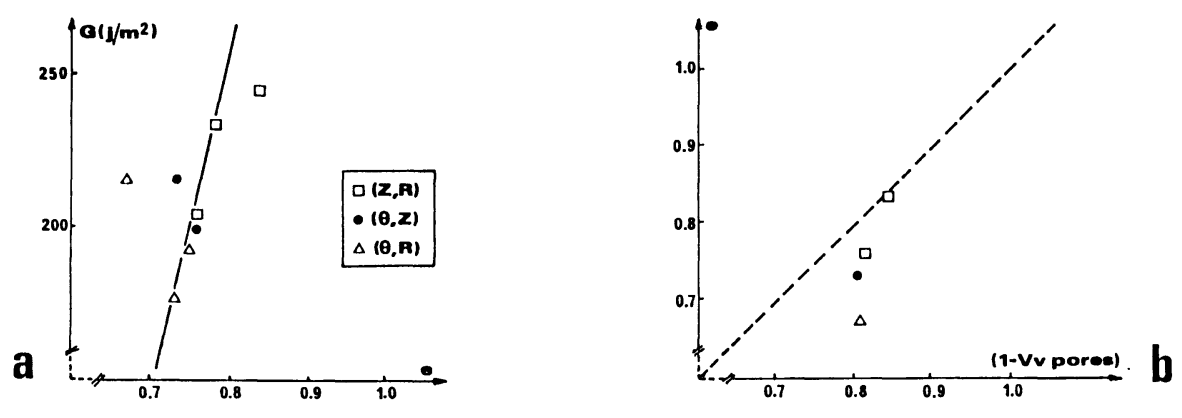

Figure 9 - Relation between the geodesic deviation and :

a) toughness

b) porosity

- there is a general good agreement between actual and simulated crack path (figure 8 ) when considering medium grain size graphite.

- the expected correlation between measured $G_{C}$ and the geodesic deviation e is good, except for one sample (figure $9 \mathrm{a}$ ). The slope of the regression from the remaining samples gives an estimation of $\gamma_{S}$ equal to $265 \mathrm{~J} / \mathrm{m}^{2}$ (with a correlation coefficient $r=0,81$ ) to be compared to the known value for dense graphite $\left(100-500 \mathrm{~J} / \mathrm{m}^{2}\right)$.

In figure $9 \mathrm{~b}$, it is shown that the geodesic deviation is always less than the matrix volume fraction (e would be equal to it for a straight line crack propagation). The range of variation of e is larger than that of the $\left(1-v_{v}\right.$ pore) parameter, as $e$ is sensitive to pore anisotropy for an almost constant pore volume fraction (see Table 2). Thus it appears that the empirical formulation connecting $\mathrm{G}_{\mathrm{c}}$ to $\mathrm{V}_{\mathrm{v}}$ pores (see relation (7)) has to be somewhat improved in further developments.

The proposed method is successful, in spite of the approximations made : two-dimensional simulations (instead of $3-D$ ones), disregard for microporosity and for small scale variations of the matrix toughness.

\section{REFERENCES}

[1] COSTER, M. et ChERMANT, J.L. "Précis d'Analyse d'Images". (Editions du C.N.R.S.) 1985.

[2] JEULIN, D. Proc. of the Sixth Pfefferkorn Conference, Niagara Fal1s (Apr. 27-May 2, 1987) (S.E.M. Inc. Publications) 1987.

[3] MATHERON, G. "Eléments pour une Théorie des Milieux Poreux". (Masson et Cie, Paris) 1967.

[4] MATIlERON, G. "Random Sets and Integral Geometry" (Wiley and Sons, New York) 1975.

[5] JEULIN, D. Thèse de Docteur-Ingénieur, Ecole des Mines de Paris, 1979.

[6] SERRA, J. "Image Analysis and Mathematical Mor.* phology" (Academic Press, London) 1982

[7] JEULIN, D. Proc. of the 5th International Symp. on Continuum Models of Discrete Systems, Nottingham (14-20 July 1985). (Publ.: A.A. Balkema, Rotterdam) 1987, p. 217.

[8] JEULIN, D. J. of Microsc. Spectrosc. Electron., Vo1. 12, $\mathrm{n}^{\circ} 1,1987, \mathrm{pp}$. 133-138.

[9] BERAN, M.J. "Statistical Continuum Theories" (Wiley-Interscience, New York) 1968. [10]BERAN, M.J., McCOY, J.J. Quant. App1. Math., 28
p. 245, 1970.

[11] KRONER, E. "Statistica1 Continuum Mechanics"

[12] KRONER, E. Linear Properties of Random Media.: the Systematic Theory. C.R., $15^{\circ}$ Colloque du Groupe Français de Rhéologie, 1980.
It is also representative of future trends in the selection of adapted morphological parameters to solve a given physical problem, deduced from the simulation of a physical process by image analysis.

\begin{tabular}{|c|c|c|c|}
\hline Orientation & $G_{C}\left(J / m^{2}\right)$ & $\begin{array}{r}\text { erage } \\
\mathrm{e} \\
\end{array}$ & $\mathrm{V}_{\mathrm{v}}$ pores \\
\hline$\theta$, & 195,7 & 0.719 & 0.17 \\
\hline$\theta, z$ & 207,9 & 0.746 & 0.177 \\
\hline $\mathrm{Z}, \mathrm{R}$ & 228.2 & 0.793 & 0.16 . \\
\hline
\end{tabular}

TABLE 2. Anisotropy of polycristalline graphite toug hness and macropore morphology.

\section{CONCLUSION}

From image analysis, morphological parameters can be measured on material samples in order to explain the changes in overall mechanical properties induced by morphological aspects on a microscopic level. 'his was illustrated in this presentation, mainly for the estimation of the strength of porous materials, but a part of the followed method, as for instance the case of crack propagation, is rather genera1.

It is expected that further progresses will become evident with the new developments of micro-mechanical models accounting for the effects of the components' morphology on the overall mechanical response of materials.

[13] KRONER, E. Statistical Modelling. In : "Mode1ling Small Deformations of Polycrystals", Ch. 8, pp. 229-291. (Elsevier Applied Science Publishers, Amsterdam) 1986.

[14] HASHIN, Z., and SHTRIKMAN, S. J. of Mech. Phys. Solids, 10, p. 343 (1962).

[15] WILLIS, J.R. Advances in Applied Mechanics, 21, 1-78 (1981).

[16] WILLIS, J.R. Journa1 of Applied Mechanics, Vo1. 105, p. 1202 (1983).

[17] JEULIN, D., STEILER, J.M. Mem. Sci. Rev. Met., 77, $\mathrm{n}^{\circ}$ 2, pp. 107-127., 1980.

[18] JEULIN, D., STEILER, J.M., DUCHENE, J.M., ISLER, D. Proc. 41st Ironmaking Conference, Pittsburgh. (Editor : A.I.M.E.) 1982.

[19] BAILEi, J.E., HILl, N.A. Proc. Brit. Ceram. Soc., n ${ }^{\circ} 15$, pp. 15-35. 1970.

[20] RICE, RW., FREIMAN, S.W., POHANKA, R.C., MECHOLSKY, J.M., WU, C.C. In : "Fracture Mechanisms of Ceramics" (Plenum Press, New York), 1977, Vo1. 4, p. 849.

[21] NIED, H.A., ARIN, K. In "Fracture Mechanics of Ceral cs" (Plenum Press, New York), 1977, Vo1. ., p. 67.

[22] JEULIN, D. Acta Stereologica, Vo1. 5, $\mathrm{n}^{\circ} 2$, pp. 233-239, 1986.

[23] PINET, M.H. Internal I.R.S.I.D. Report, 1984.

[24] LARQUETOUX, G. Internal I.R.S.I.D. Report, 1985.

[25] LANTUEJOUL, C. , BEUCHER, S., J. of Microscopy, Vol. 121 Pt. 1, pp. 39-49, 1980. 